\title{
Research Article \\ Impact of Daily Exercise Compared to Exercise on Alternating Days on Post-Exercise Blood Pressure Reduction in Men with Elevated Blood Pressure
}

\author{
Zachary Zeigler ${ }^{1, *}$, Malachi Votaw ${ }^{1}$, Connor Dreos $^{1}$, Lydia Durnil $^{1}$, Jamie Terran${ }^{1}$, Danielle Akin , \\ Trevor Nordin ${ }^{1}$
}

'Grand Canyon University, MESA, United States.

Article Information
History:
Received: March 23, 2018
Accepted: Jun 5, 2018
Published: Jun 14, 2018
Keywords:
Acute exercise
Daily exercise
Elevated blood pressure
Hypertension
Post exercise hypotension

\begin{abstract}
Objectives: Acute exercise can result in post-exercise hypotension (PEH) lasting up to 24-h. Whether exercise performed on consecutive days would lead to an accumulating PEH effect has yet to be determined. The purpose of this study was to compare daily exercise (DE) to exercise performed on alternating days (AE) and control (CON) on PEH.

Methods: Sedentary men 18-30 yr with elevated blood pressure (BP) participated in this three-arm randomized cross-over trial. The primary comparison was PEH between three groups (CON, $A E, D E$ ) over time (day $1,2,3)$. Both exercise groups were prescribed the same exercise intensity $(70-75 \% \mathrm{HRmax})$, and total duration of exercise $(90 \mathrm{~min})$ on a cycle ergometer. DE performed exercise on three consecutive days (three bouts $30 \mathrm{~min}$ ). AE performed exercise on two alternating days ( 2 bouts $45 \mathrm{~min}$ ). Following exercise subjects remained in the laboratory for 1 -h $\mathrm{h}$ while BP was taken every 5 -min.
\end{abstract}

Results: Nine overweight $\left(\mathrm{BMI}=29.2 \pm 4.5 \mathrm{~kg} / \mathrm{m}^{2}\right)$, young $(22.7 \pm 2.4$ years $)$, moderately fit (VO2pe$\mathrm{ak}=35.6 \pm 7.3 \mathrm{ml} . \mathrm{kg} . \mathrm{min})$, male subjects with elevated BP $(126.2 \pm 10.4$ and $73.3 \pm 6.4 \mathrm{mmHg})$ completed the study. There was a significant systolic BP condition by day effect such that on day three systolic BP (CON 119.0 \pm 9.3, AE 118.9 \pm 15.0 , DE $115.0 \pm 11.9 \mathrm{mmHg}$ ), and diastolic BP (CON 71.9 $\pm 6.6, A E 68.4 \pm 10.3$, $\mathrm{DE} 67.6 \pm 6.2 \mathrm{mmHg}$ ) were lowest during the post-exercise $\mathrm{DE}$ condition $(\mathrm{P}<0.001)$. Additionally, $\mathrm{DE}$ saw a significant reduction of resting systolic BP between day 1 and day $4(122.8 \pm 10.2 \mathrm{mmHg}$ vs $113.1 \pm 12.0 \mathrm{mmHg} ; \mathrm{P}=0.022$, respectively) and a borderline significant reduction between day 1 and day $3(122.8 \pm 10.2 \mathrm{mmHg}$ vs $114.8 \pm 10.0 \mathrm{mmHg} P=0.051$, respectively). $D E$ saw a borderline significant resting diastolic $B P$ reduction between day 1 and day $3(73.2 \pm 7.2 \mathrm{mmHg}$ vs $68.6 \pm 6.5 \mathrm{mmHg} P=0.058$, respectively) and a significant reduction between day 1 and day $4(73.2 \pm 7.2 \mathrm{mmHg}$ vs $66.4 \pm 4.3 \mathrm{mmHg}$; $\mathrm{P}=0.022$, respectively).

Conclusions: In conclusion, the post-exercise BP lowering effect of the prior exercise session appeared to accumulate during DE such that day 3 was the lowest of all conditions and days.

\section{INTRODUCTION}

According to the World Health Organization, more than a third of the world's deaths can be attributed to a small number of risk factors. Among the top five leading risk factors are hypertension (HTN), obesity and physical inactivity [1]. Based off the recent blood pressure (BP) guideline

*Correspondence: Zachary S. Zeigler, College of Science, Engineering, and Technology, Grand Canyon University,

3300 W Camelback Rd, MESA, United States.

E-mail address: Zachary.zeigler@gcu.edu changes roughly $46 \%$ of Americans are now classified as HTN [2]. Approximately 7.1 million deaths worldwide are attributed to HTN [3], and lifetime risk for developing HTN is estimated at $90 \%$ [4].

The recent BP guidelines also emphasized an "at risk" BP classification being titled "elevated Blood Pressure" (Systolic BP (SBP) 120-129 and Diastolic BP (DBP) < 80 mmHg) [2]. Those with elevated $\mathrm{BP}$ are the most vulnerable population for developing HTN [5], and are at increased risk for cardiovascular morbidity and mortality [6]. However, the only recommended treatment for those classified in the category of elevated BP is lifestyle modification, including exercise terms of the Creative Commons Attribution License (http://creativecommons.org/licenses/by/4.0/), which permits unrestricted use, distribution, and reproduction in any medium, provided the original work is properly cited. 
interventions [6].

Exercise training has shown to reduce BP burden [7]. Indeed, even an acute exercise bout can result in post-exercise hypotension (PEH) lasting up to 24-h, measured by 24-h ambulatory BP, displaying the possibility of merging into a following exercise session when conducted daily [810]. Thus, acute and chronic exercise BP response cannot be viewed in isolation. Acute post-exercise BP lowering responses may accrue in a cumulative manner so that following subsequent sessions an added post-exercise lowering BP effect may be seen [11]. The BP lowering impact of acute exercise could possibly explain exercise training $\mathrm{BP}$ reductions, thus highlighting the significance of the acute exercise response [11-13]. Additionally, prior research has shown an additive effect of consecutive exercise on glycemic control [14] and triglycerides [15]. The current study adds to the body of literature by suggesting that the well-established post-exercise BP reduction may accumulate when daily exercise (DE) is conducted to produce superior BP lowering effects. Additionally, if an accumulation effect is seen, it is unknown if DE is superior to exercise performed on alternating days (AE).

Investigations designed to compare different components of the exercise prescription for those with elevated BP are lacking [16]. It has been suggested that the current recommendation on training frequency should be changed from accumulating $30 \mathrm{~min}$ on most, preferably all days of the week, to simply all days of the week [16].

The purpose of this study was to assess the possible additive effects of DE compared to $\mathrm{AE}$ on $\mathrm{PEH}$ in males with elevated BP. It is hypothesized that when DE is performed an additive effect will be witnessed such that PEH on day 2 and 3 will be greater than the PEH on day 1 .

\section{METHODS}

\section{Study Design}

The present study was a three-arm randomized experimen- tal design. The Grand Canyon Institutional Review Board approved this study (IRB \# 948803-2). The primary comparison was the magnitude of $\mathrm{PEH}$ between the three groups and within each group over time. Figure 1 gives graphical depiction of the research design. This cross-over experimental trial consisted of subjects randomized into 3 groups: DE, $\mathrm{AE}$, and a non-exercise control (CON). Additionally, after completion of the first condition subjects were randomly assigned to the last two conditions and all subjects completed each condition. A period of one week was used as the washout time, thus, each condition was performed one week apart. Exercise duration between groups were matched at $90 \mathrm{~min}$ split into either three $30 \mathrm{~min}$ sessions (DE), or two $45 \mathrm{~min}$ sessions (AE). Exercise was performed on a cycle ergometer at an intensity of $70-75 \%$ heart rate (HR) max. Blood pressure was measured at $5 \mathrm{~min}$ intervals for $1 \mathrm{~h}$ following each exercise session.

\section{Participants and Settings}

\section{a. Inclusion Criteria}

Nine, sedentary (not meeting physical activity guidelines of $150 \mathrm{~min} /$ week determined by the International Physical Activity Questionnaire [17], men aged 18-30 yr with elevated BP (SBP 120-129 mmHg AND DBP <80 mmHg) were recruited to participate in this experimental trial.

\section{b. Exclusion Criteria}

Having known cardiovascular, pulmonary, renal or metabolic disease, and current smokers, were excluded from the study. In addition, those who are morbidly obese (BMI $>40$ $\mathrm{kg} / \mathrm{m}^{2}$ ) or had HTN (SBP $>140 \mathrm{~mm} \mathrm{Hg}$ or DBP $>90 \mathrm{~mm}$ $\mathrm{Hg}$ ) were excluded. Subjects were also excluded if they were taking oral anti-histamine medications as it is known that the histaminergic pathways are responsible for much of the $\mathrm{PEH}$ response [18].

\section{c. Consent Process}

The informed consent was given to potential participants

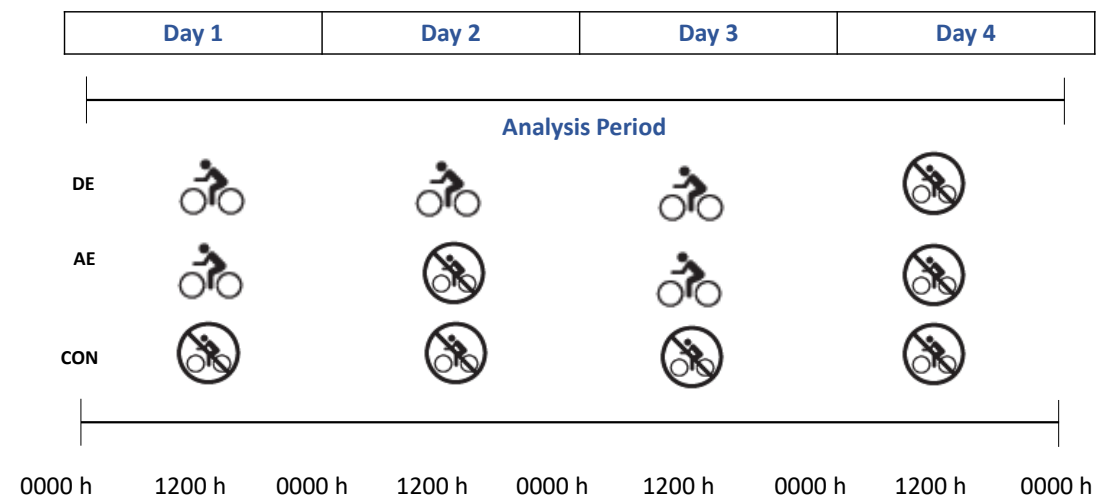

Figure 1. Schematic overview of the experimental period.

Subjects completed each condition, non exercise (CON), 90 min of cycling at $75 \%$ of HR max distributed as two 45 min sessions on alternating days (AE), and three $30 \mathrm{~min}$ sessions performed daily (DE). Following exercise, or rest during CON, subjects remained in the lab for $60 \mathrm{~min}$. During the post-exercise period, subjects' BP was measured every 5 min. 
and adequate time was allowed for them to read over it. Once the potential participant was finished reading the informed consent, the investigator gave the individual an opportunity to ask any questions.

\section{BP Screening and Baseline Measurements}

For all participants, three BP measurements were taken on two separate occasions $3 \mathrm{~d}$ apart according to the protocol described by the World Health Organization [3]. On the first day BP was taken in both arms. The arm with the highest BP was used for screening on the second day. Six total measurements were averaged together and used for determining if subjects met the elevated BP criteria. Blood pressure was measured via the digital BP device (Connex ${ }^{\circledast}$ ProBP $^{\mathrm{Tm}} 3400$, Welch Allyn, USA).

Once subjects met the inclusion criteria, baseline measurements were assessed. Blood pressure was first assessed in a seated resting condition in the laboratory before the first study day. Following BP measurement body composition was measured and then a $\mathrm{VO}_{2 \text { peak }}$ test was performed (see below for procedures).

\section{a. Body Composition}

Body composition was measured via whole body air displacement plethysmography (Bod Pod). Subjects were asked to wear a bathing suit or tight-fitting shorts and to remove their shirt for testing and remove all jewelry. Subjects were first weighed on a scale in bare feet. Subjects were then asked to place a cap on their head to cover their hair. Subjects then sat in the Bod Pod for 2-3 measurements of $50 \mathrm{sec}$ each. Following the Bod Pod the $\mathrm{VO}_{2 \text { peak }}$ test was conducted.

\section{b. $\mathrm{VO}_{2 \text { peak }}$}

This test was a ramp cycle ergometer protocol. Subjects began on the cycle ergometer at 25 watts for $5 \mathrm{~min}$ for a warmup. Every min the wattage increased by 20 watts until the subject reached volitional failure or requested to stop. Subjects were given a $5 \mathrm{~min}$ cool down period on the ergometer at 25 watts. Oxygen consumption was analyzed continuously by the Vmax metabolic cart (CareFusion, Franklin Lakes, $\mathrm{NJ}$ ) and averaged every 10 seconds. $\mathrm{VO}_{2 \text { peak }}$ was determined as the 2 highest consecutive 10 -second oxygen consumption values.

\section{Measurement Procedures}

Subjects were asked to consume the same foods over the 4-d measurement period of each condition and a dietary food $\log$ was given to aid in this endeavor. Subjects were instructed to refrain from any physical activity for 72-h prior to initiating the study. Baseline measurements were assessed prior to any experimental visits. Laboratory visits were conducted at approximately $1230 \mathrm{~h}$ because of the superior effect that afternoon exercise has compared to morning exercise on PEH [19]. When subjects arrived at the laboratory they were asked to sit quietly for 20-min.
Two BP readings were taken to ensure hemodynamic stability. Following this verification of hemodynamic stability, two additional BP readings were then taken and averaged together to give a "pre-exercise" BP value. After the completion of exercise or a non-exercise CON, subjects were asked to remain in the laboratory for 1-h while laboratory BP was taken every 5-min for a total of 12 post exercise BP measurements. During CON, subjects remained in the laboratory for roughly $1.5 \mathrm{~h}$ while resting $\mathrm{BP}$ and the "post-exercise" measurements were taken. All BP measurements were made while the subjects were sitting in an upright position.

\section{Exercise Protocol}

Exercise during both $\mathrm{DE}$ and $\mathrm{AE}$ were prescribed the same intensity (70-75\% of measured HR max), and total duration of exercise (90 $\mathrm{min})$. Exercise was performed on a cycle ergometer. The only difference between the two exercise conditions was that during DE, exercise was conducted on three consecutive days (three bouts of $30 \mathrm{~min}$ ) compared to during $\mathrm{AE}$ where exercise was conducted on two alternating days (2 bouts of $45 \mathrm{~min}$ ). Subjects were required to perform exercise in the laboratory under supervision of one of the researchers. To ensure exercise intensity adherence, HR monitors were worn by the subjects and researchers monitored HR every 5 min during the exercise sessions.

\section{Statistical Analysis}

Data were analyzed using SPSS software (version 24). The alpha error for the primary end point was set at 0.05 and the beta error level at 0.2 . Data are expressed as mean \pm standard deviation (SD). All $\mathrm{P}$ values were calculated assuming two-tailed hypothesis; $\mathrm{P}<0.05$ was considered statistically significant. Data were analyzed for normality and transformed if necessary. Descriptive statistics were used for the demographics of the subjects. Resting BP within group (CON, DE, AE) over time (Day 1, Day 2, Day 3, Day 4) and between group over time was analyzed using Linear Mixed Models (LMM) to detect differences in systolic and diastolic BP. PEH was analyzed by the average of the 12 post-exercise $\mathrm{BP}$ values, or in case of the CON condition it was following rest, was compared within group (CON, $\mathrm{DE}, \mathrm{AE}$ ) over time (Day 1, Day 2, Day 3). This comparison was used to assess a possible accumulating impact of DE. Overall effect was compared using LMM. Additionally, the pre-exercise resting $\mathrm{BP}$ values were compared to the post exercise BP values of the same day via LMM to assess the impact of pre-exercise BP on same day PEH. LMM was conducted in a hierarchical fashion using Restricted Maximum Likelihood model and 'variance components' covariance error structure. Both fixed and random effects were explored in the model. Sample size was calculated using a 2 -sided hypothesis $\alpha=0.05$ and $\beta=0.20$ based on previous work provided effect size estimates [10]. In response to an acute bout of aerobic exercise using within subjects 
Table 1. Demographics $(\mathrm{N}=9)$

\begin{tabular}{ll}
\hline & Mean \pm SD \\
\hline Height $(\mathrm{cm})$ & $175.9 \pm 3.7$ \\
Weight $(\mathrm{kg})$ & $76.5 \pm 11.3$ \\
BMI $\left(\mathrm{kg} / \mathrm{m}^{2}\right)$ & $29.2 \pm 4.5$ \\
Body Fat $(\%)$ & $27.9 \pm 6.5$ \\
Age $(\mathrm{y})$ & $22.7 \pm 2.4$ \\
$\mathrm{VO}_{2}$ peak $\left(\mathrm{mL} \cdot \mathrm{kg}^{-1} \cdot \mathrm{min}^{-1}.\right)$ & $35.6 \pm 7.3$ \\
\hline
\end{tabular}

differences, BP has shown to be reduced following exercise at a magnitude of $3 / 2 \mathrm{mmHg}$ with a SD of $\pm 1 \mathrm{mmHg}$ [10] when compared to a non-exercise control period covering the same time span and within the same subject. Eight total subjects were needed to properly power the current study.

\section{RESULTS}

Nine subjects completed each stage of the study. Table 1 presents demographic characteristics of the subjects. Briefly, subjects were relatively young, overweight, and on the lower end of the fitness spectrum for their age [20].

\section{Resting BP Changes}

Resting pre-exercise BP values between days 1 and days 3, in addition to resting BP on day 4 were compared to assess the impact of exercise the day before on resting BP. Figure 2 (SBP) and Figure 3 (DBP) illustrate there were no significant differences in resting SBP $(\mathrm{P}=0.874)$ or $\mathrm{DBP}(\mathrm{P}=0.459)$ for CON over days 1 through 4 . There was a non-significant trend for AE to produce decreased resting SBP between days 1 through $3(\mathrm{P}=0.079)$. However, there was a significant reduction of resting SBP between day 1 and day 4 during $\mathrm{AE}(125.0 \pm 11.3 \mathrm{~mm} \mathrm{Hg}$ vs $119.3 \pm 13.4 \mathrm{~mm} \mathrm{Hg} ; \mathrm{P}=0.016$, respectively). Resting DBP during AE only significantly reduced between days 1 and $4(70.2 \pm 6.4 \mathrm{~mm} \mathrm{Hg}$ vs $64.5 \pm$ $8.6 \mathrm{~mm} \mathrm{Hg} ; \mathrm{P}<0.001$, respectively). DE saw a significant reduction of resting SBP between day 1 and day 4 (122.8 \pm $10.2 \mathrm{~mm} \mathrm{Hg}$ vs $113.1 \pm 12.0 \mathrm{mmHg}$; $=0.022$, respectively) and a borderline significant reduction between day 1 and day $3(122.8 \pm 10.2 \mathrm{mmHg}$ vs $114.8 \pm 10.0 \mathrm{mmHg} ; \mathrm{P}=0.051$, respectively). DE saw a borderline significant resting DBP

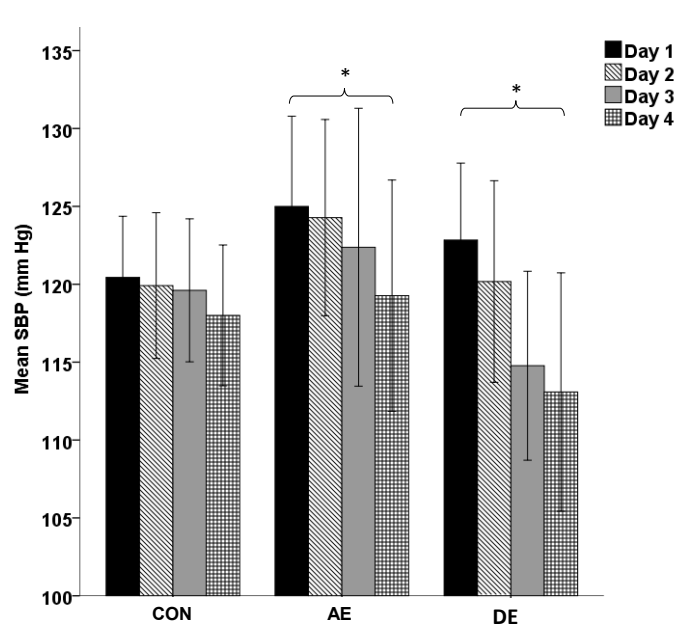

Figure 2. Resting SBP values compared between days 1 through 4 within each condition.

* represents $P<0.05$

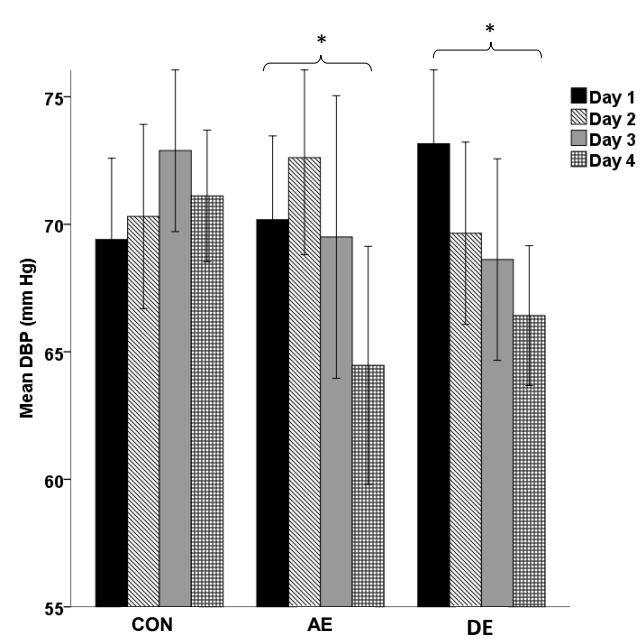

Figure 3. Resting DBP values compared between days 1 through 4 within each condition.

* represents $P<0.05$

Table 2. Average PEH data within condition over time

\begin{tabular}{|c|c|c|c|c|}
\hline & Day 1 & Day 2 & Day 3 & P-value \\
\hline \multicolumn{5}{|c|}{$\mathrm{SBP}(\mathrm{mm} \mathrm{Hg})$} \\
\hline $\mathrm{CON}$ & $120.3 \pm 10.4$ & $120.9 \pm 11.5$ & $119.0 \pm 9.3$ & 0.434 \\
\hline $\mathrm{AE}$ & $124.1 \pm 13.1^{\mathrm{a}}$ & $124.2 \pm 14.1^{\mathrm{a}}$ & $118.9 \pm 15.0^{\mathrm{b}}$ & $<0.015$ \\
\hline $\mathrm{DE}$ & $121.5 \pm 12.3^{\mathrm{a}}$ & $119.1 \pm 11.1^{\mathrm{a}}$ & $115.0 \pm 11.9^{\mathrm{b}}$ & $<0.001$ \\
\hline \multicolumn{5}{|c|}{ DBP (mm Hg) } \\
\hline $\mathrm{CON}$ & $70.2 \pm 7.7$ & $71.8 \pm 8.0$ & $71.9 \pm 6.6$ & 0.79 \\
\hline $\mathrm{AE}$ & $70.6 \pm 5.6$ & $71.8 \pm 9.3^{\mathrm{a}}$ & $68.4 \pm 10.3^{b}$ & 0.017 \\
\hline $\mathrm{DE}$ & $71.7 \pm 7.1^{\mathrm{a}}$ & $68.7 \pm 7.0^{\mathrm{b}}$ & $67.6 \pm 6.2^{\mathrm{b}}$ & $<0.001$ \\
\hline
\end{tabular}

Differing letters denote statistically different from each other at a $P<0.05$. 


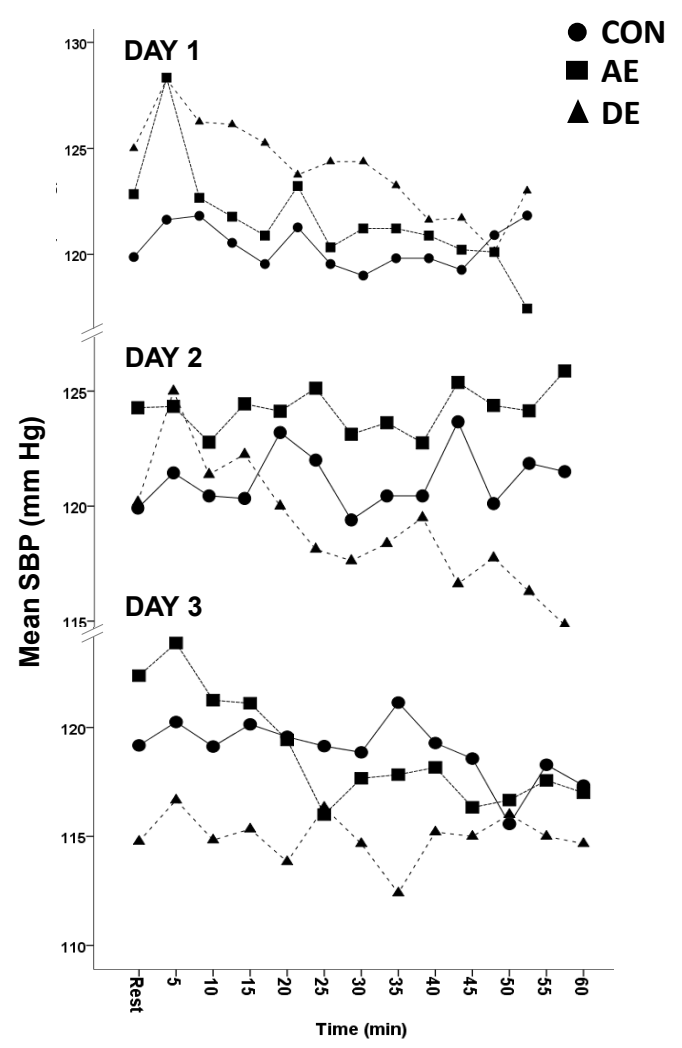

Figure 4. Pre and post-exercise SBP measured every 5-min for one hour following exercise or rest.

reduction between day 1 and day $3(73.2 \pm 7.2 \mathrm{mmHg}$ vs $68.6 \pm 6.5 \mathrm{mmHg} ; \mathrm{P}=0.058$, respectively) and a significant reduction between day 1 and day $4(73.2 \pm 7.2 \mathrm{mmHg}$ vs 66.4 $\pm 4.3 \mathrm{mmHg} ; \mathrm{P}=0.022$, respectively).

\section{Post exercise BP compared to Day 1}

Post-exercise BP was compared within condition over time with the primary comparison being days 2 and 3 compared to day 1 . This allowed for the assessment of a possible accumulation effect. Table 2 shows average post-exercise BP (average of the 12 post-exercise, or post rest during CON, measurements) values for each condition (CON, DE, AE) over time (days 1, day 2, day 3). There was no significant post-exercise $\mathrm{BP}$ difference for CON over the 3-day measurement period for either SBP $(\mathrm{P}=0.434)$ or DBP $(\mathrm{P}$ $=0.790)$. There was no significant SBP post-exercise BP difference between days 1 and 2 during AE. Days 1 and 2 did however significantly differ from day 3 during $\mathrm{AE}(\mathrm{P}=$ $0.015)$. Day 3 during $A E$ was the lowest for $\mathrm{DBP}(\mathrm{P}=0.017)$. During DE, day 3 was the lowest post-exercise $\mathrm{BP}$ and was significantly lower than days 1 and $2(\mathrm{P}<0.001)$. Both days 2 and 3 were significantly lower than day 1 during $\mathrm{DE}$ for DBP $(\mathrm{P}<0.001)$.

\section{PEH compared to Pre-exercise Resting BP}

Post-exercise hypotension was compared between the

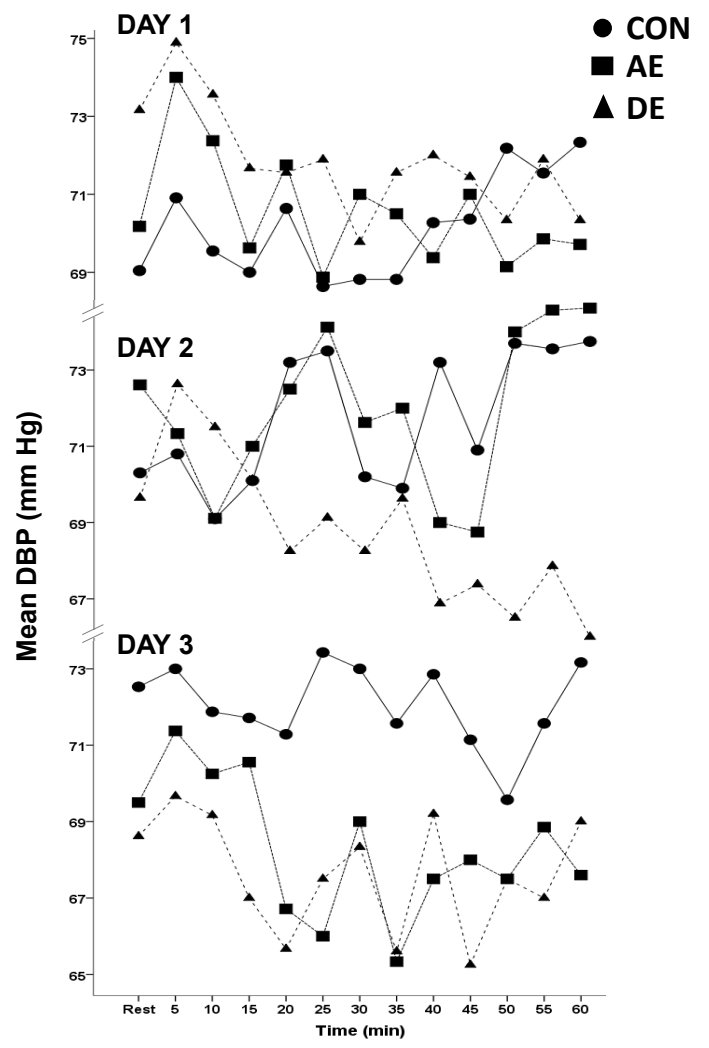

Figure 5. Pre and post-exercise DBP measured every 5-min for one hour following exercise or rest.

pre-exercise resting $\mathrm{BP}$ values and the average post-exercise values of the same day. Figure 4 (SBP) and Figure 5 (DBP) show $\mathrm{PEH}$ compared to pre-exercise BP values within group over time. The CON saw no post-exercise SBP or $\mathrm{DBP}$ reductions on day 1 , day 2 or day 3 (all $\mathrm{P}>0.05$ ). AE saw no post-exercise SBP or DBP reduction on day $1(\mathrm{P}=$ $0.534, \mathrm{P}=0.518$, respectively $)$ or day $2(\mathrm{P}=0.656, \mathrm{P}=0.517$, respectively). AE saw a significant $\mathrm{SBP}$ reduction following exercise on day $3(-5.3 \pm 5.4 \mathrm{~mm} \mathrm{Hg}, \mathrm{P}=0.004)$ with no significant change in DBP $(\mathrm{P}=0.247)$. There was not a significant $\mathrm{DBP}$ reduction following exercise during $\mathrm{AE}$ on day $3(\mathrm{P}=0.247)$. $\mathrm{DE}$ on day 1 , day 2 , and day 3 did not cause a reduced SBP or DBP following exercise (all P $<0.05$ ).

\section{DISCUSSION}

In summary, our data showed that resting SBP and DBP was the lowest on day 4 of the DE condition. Additionally, the average SBP PEH values were the lowest following exercise on day 3 for the $\mathrm{DE}$ condition. In fact, the only condition in which post-exercise SBP was significantly lower than the CON condition was day 3 during DE. Taken together this suggests that the impact of exercise on the day or days prior indeed did accumulate to produce a pronounced $\mathrm{BP}$ lowering effect.

Our hypothesis was formulated based on prior research showing an additive effect of consecutive exercise on glycemic control [14] and triglycerides [15]. Additionally, it has 
been shown that PEH may last for 24-h $[8,9,21]$, displaying the possibility of merging into a following exercise session when conducted daily. The current study adds to the body of literature by suggesting that the acute BP lowering impact may accumulate when DE is conducted to produce superior BP lowering effects.

Prior research examining impact of DE on BP had limitations. Nelson et al evaluated the effects of exercise frequency on BP and found that the decrease in BP in HTN subjects was significantly greater on a seven- as opposed to three-times per week exercise program. However, because the total amount of exercise was $135 \mathrm{~min} / \mathrm{wk}$ and $315 \mathrm{~min} /$ wk in the three and seven times per week group, respectively, the effects of exercise duration could not be isolated from frequency. Other training studies have attempted to answer the question of the necessity of DE but did not assess the acute BP lowering affect $[22,23]$. The current study matched exercise intensity and duration between exercise performed on three consecutive days and exercise on two alternating days allowing for the direct assessment of the impact of DE on $\mathrm{PEH}$ and resting $\mathrm{BP}$ outcomes.

The current study also found that post-exercise SBP and $\mathrm{DBP}$ for AE was lower on day 3 (exercise did not occur on day 2) when compared to day 1 . This suggests that the BP lowering impact of exercise on day 1 persisted for $48 \mathrm{~h}$. This adds to the theory that chronic exercise training effects on BP may merely reflect the acute $\mathrm{PEH}$ phenomenon with sustained BP reductions accumulating from each bout of exercise [24]. Indeed the magnitude of $\mathrm{PEH}$ to an acute bout is a strong predictor of how well one may respond to a chronic exercise training program [25-27]. In fact, when analyzed separately it has been shown that there is no difference in magnitude of BP (24-h ambulatory BP) reduction between the acute and chronic response from exercise $[21,28]$.

It is not readily apparent as to why BP during DE was not lower following exercise on days 1 and 2 when compared to CON. It could be that our exercise intensity was not high enough to illicit a response. Although controversial, recent data on exercise intensity and $\mathrm{PEH}$ has suggested that the higher intensity the exercise the greater the magnitude of $\mathrm{BP}$ reduction [29]. Our study also found that the longer duration of the $\mathrm{AE}$ sessions did not provoke greater $\mathrm{PEH}$ or greater resting BP reductions when compared to the shorter DE condition. This supports previous work that suggests $\mathrm{PEH}$ is a low threshold phenomenon in terms of the duration of exercise needed [30]. This is relevant when considering that time is one of the biggest deterrents of exercise engagement.

Only post-exercise SBP on day 3 during AE was lower than pre-exercise resting values. It has been determined that one of the biggest predictors of $\mathrm{PEH}$ is resting pre-exercise values [31]. Resting SBP pre-exercise value on day 3 was significantly lower during DE when compared to AE. This resting SBP difference more than likely explains why DE during day 3 did not lower SBP when compared to the pre-exercise SBP value. In fact, it appeared that the lower- ing of the pre-exercise BP value negated almost all the $\mathrm{PEH}$ impact that was expected to be seen. This study adds to the literature by providing further support that pre-exercise BP values are a predictor of the magnitude of the PEH effect.

Our study has its limitations. First, our population was relatively young, healthy, and active. Divergent results may be seen in diseased or older populations. Also, subjects were free to eat on their own and eat what they liked. Subjects were asked to eat the same thing before coming to the lab and between conditions and a dietary recall was given to aid in this endeavor. Additionally, the post-exercise duration was only for one hour, thus minimizing generalizability. This time frame was chosen however due to past data suggesting the lowest post-exercise BP value takes place within the first hour $[32,33]$ and because of prior data suggesting that this immediate reduction could possible predict training responses [25-27]. Exercise duration for a single exercise session was not matched. However, BP lowered in response to the shorter DE to a greater degree than the longer AE session. Suggesting that even if the longer durations could be superior, the magnitude was not great enough to overpower the importance of back to back exercise sessions. Additionally, outside physical activity was not closely monitored. However, subjects were asked to not engage in any outside activity above what was prescribed by the researchers. Lastly, only males were recruited for this study. However, men were chosen due to the lack of research within this population and due to possible menstrual effects on the vasculature. Additionally, data suggests sex is not a major player in the post exercise BP response [34].

There are many strengths to our study. Our cross-over study design increases intra-subject control and decreases bias. We also made sure to time match the different conditions to control for diurnal variations. A flaw in $\mathrm{PEH}$ research is seen when the primary comparison is resting pre-exercise values to post exercise values of the same day. BP changes on a diurnal basis and this diurnal change could explain the $\mathrm{PEH}$ effect opposed to the previous exercise activity. Thus, we compared $\mathrm{PEH}$ between a time matched control in addition to pre-exercise values. We also used the public exercise guidelines of moderate intensity exercise in 30 min bouts. Lastly, we matched total exercise duration for each condition to better isolate the impact of exercise frequency.

It should be noted that upwards of $25 \%$ of people with the goal of lowering BP in response to exercise have been classified as "non-responders" [35]. The current study showed that post-exercise BP did not reach statistical significant reductions until day 3 when compared to CON. Those classified as "non-responders" may have not yet been exposed to enough of an exercise dose. This should be taken under consideration when labeling subjects as "non-responders".

From a clinical standpoint, the BP reductions seen in the current study are not negligible. Indeed reductions of just $5 \mathrm{mmHg}$ of systolic BP in the population would result in a $14 \%$ overall reduction in mortality due to stroke, a $9 \%$ re- 
duction in mortality due to $\mathrm{CHD}$, and a $7 \%$ decrease in allcause mortality [36].

\section{CONCLUSIONS}

In conclusion, DE appeared to cause a BP lowering accumulating impact such that BP was the lowest after exercise was conducted three days in a row. The results of this study should be used to tailor future research using training protocols designed to test ideal exercise frequency to lower BP.

\section{ACKNOWLEDGEMENTS}

The authors would like to thank the participants and the undergraduate students who helped in data collection for this work.

\section{Conflicts of Interest}

The authors declare no conflict of interest.

\section{REFERENCES}

1. World Health Organization. Global Health Risks: mortality and burden of disease attributable to selected major risks. 2009. http://www.who.int/healthinfo/global_burden_disease/global_health_risks/en/. (Accessed Feb 1, 2017).

2. Whelton PK, Carey RM, Aronow WS, et al. 2017 ACC/ AHA/AAPA/ABC/ACPM/AGS/APhA/ASH/ASPC/ NMA/PCNA guideline for the prevention, detection, evaluation, and management of high blood pressure in adults: a report of the American College of Cardiology/American Heart Association Task Force on Clinical Practice Guidelines. Ann Intern Med. 2018;168(5):351358.

3. Whitworth JA, World Health Organization, International Society of Hypertension Writing Group. 2003 World Health Organization (WHO)/International Society of Hypertension (ISH) statement on management of hypertension. J Hypertens. 2003;21(11):1983-92.

4. Vasan RS, Beiser A, Seshadri S, et al. Residual lifetime risk for developing hypertension in middle-aged women and men: The Framingham Heart Study. JAMA. 2002;287(8):1003-10.

5. Landsberg L, Aronne LJ, Beilin LJ, et al. Obesity-related hypertension: pathogenesis, cardiovascular risk, and treatment--a position paper of the The Obesity Society and The American Society of Hypertension. Obesity (Silver Spring). 2013;21(1):8-24.

6. Chobanian AV, Bakris GL, Black HR, et al. Seventh report of the Joint National Committee on Prevention, Detection, Evaluation, and Treatment of High Blood Pressure. Hypertension. 2003;42(6):1206-52.

7. Pescatello LS, Franklin BA, Fagard R, et al. American College of Sports Medicine position stand. Exercise and hypertension. Med Sci Sports Exerc. 2004;36(3):533-53.

8. Angadi SS, Weltman A, Watson-Winfield D, et al. Effect of fractionized vs continuous, single-session exercise on blood pressure in adults. J Hum Hypertens. 2010;24(4):300-2.

9. Bhammar DM, Angadi SS, Gaesser GA. Effects of fractionalized and continuous exercise on 24-h ambulatory blood pressure. Med Sci Sports Exerc. 2012;44(12):227076.

10. Pescatello LS, Kulikowich JM. The aftereffects of dynamic exercise on ambulatory blood pressure. Med Sci Sports Exerc. 2001;33(11):1855-61.

11. Haskell WL. J.B. Wolffe Memorial Lecture. Health consequences of physical activity: understanding and challenges regarding dose-response. Med Sci Sports Exerc. 1994;26(6):649-60.

12. Thompson PD, Crouse SF, Goodpaster B, Kelley D, Moyna N, Pescatello L. The acute versus the chronic response to exercise. Med Sci Sports Exerc. 2001;33(6):S438-45.

13. Seals DR, Silverman HG, Reiling MJ, Davy KP. Effect of regular aerobic exercise on elevated blood pressure in postmenopausal women. Am J Cardiol. 1997;80(1):4955.

14. Praet SF, van Loon LJ. Optimizing the therapeutic benefits of exercise in Type 2 diabetes. J Appl Physiol (1985). 2007;103(4):1113-20.

15. Gyntelberg F, Brennan R, Holloszy JO, Schonfeld G, Rennie MJ, Weidman SW. Plasma triglyceride lowering by exercise despite increased food intake in patients with type IV hyperlipoproteinemia. Am J Clin Nutr. 1977;30(5):716-20.

16. Pescatello LS, Franklin BA, Fagard R, et al. American College of Sports Medicine position stand. Exercise and hypertension. Med Sci Sports Exerc. 2004;36(3):533-53.

17. Rosenberg DE, Bull FC, Marshall AL, Sallis JF, Bauman AE. Assessment of sedentary behavior with the International Physical Activity Questionnaire. J Phys Act Health. 2008;5(S1):S30-44.

18. Halliwill JR, Buck TW, Lacewell AN, Romero SA. Postexercise hypotension and sustained postexercise vasodilatation: what happens after we exercise. Exp Physiol. 2012;98(1):7-18.

19. Jones H, Pritchard C, George K, Edwards B, Atkinson $\mathrm{G}$. The acute post-exercise response of blood pressure varies with time of day. Eur J Appl Physiol. 2008;104(3):481-9.

20. Shvartz E, Reibold RC. Aerobic fitness norms for males and females aged 6 to 75 years: a review. Aviat Space Environ Med. 1990;61(1):3-11.

21. Pescatello LS, Kulikowich JM. The aftereffects of dynamic exercise on ambulatory blood pressure. Med Sci Sports Exerc. 2001;33(11):1855-61.

22. Ishikawa-Takata K, Ohta T, Tanaka H. How much exercise is required to reduce blood pressure in essential hypertensives: a dose-response study. Am J Hyperten. 2003;16(8):629-33.

23. Miyatake N, Takahashi K, Wada J, et al. Daily exercise 
lowers blood pressure and reduces visceral adipose tissue areas in overweight Japanese men. Diabetes Res Clin Pract. 2003;62(3):149-57.

24. Hamer M. The anti-hypertensive effects of exercise. Sports Med. 2006;36(2):109-16.

25. Hecksteden A, Grutters T, Meyer T. Association between postexercise hypotension and long-term training-induced blood pressure reduction: a pilot study. Clin J Sport Med. 2013;23(1):58-63.

26. Liu S, Goodman J, Nolan R, Lacombe S, Thomas SG. Blood pressure responses to acute and chronic exercise are related in prehypertension. Med Sci Sports Exerc. 2012;44(9):1644-52.

27. Kiviniemi AM, Hautala AJ, Karjalainen JJ, et al. Acute post-exercise change in blood pressure and exercise training response in patients with coronary artery disease. Front Physiol. 2015;5:526.

28. Jennings GL, Deakin G, Korner P, Meredith I, Kingwell B, Nelson L. What is the dose-response relationship between exercise training and blood pressure? Ann Med. 1991;23(3):313-8.

29. Pescatello LS, MacDonald HV, Ash GI, et al. Assessing the existing professional exercise recommendations for hypertension: a review and recommendations for future research priorities. Mayo Clin Proc. 2015;90(6):801812.

30. Pescatello LS, MacDonald HV, Lamberti L, Johnson BT. Exercise for hypertension: a prescription update integrating existing recommendations with emerging re- search. Curr Hypertens Rep. 2015;17(11):87.

31. MacDonald JR. Potential causes, mechanisms, and implications of post exercise hypotension. J Hum Hypertens. 2002;16(4):225-36.

32. Somers VK, Conway J, Coats A, Isea J, Sleight P. Postexercise hypotension is not sustained in normal and hypertensive humans. Hypertension. 1991;18(2):211-5.

33. Angadi SS, Bhammar DM, Gaesser GA. Postexercise Hypotension After Continuous, Aerobic Interval, and Sprint Interval Exercise. J Strength Cond Res. 2015;29(10):2888-93.

34. Brito L, Queiroz A, Forjaz C. Influence of population and exercise protocol characteristics on hemodynamic determinants of post-aerobic exercise hypotension. Braz J Med Biol Res. 2014;47(8):626-36.

35. Bouchard C, Rankinen T. Individual differences in response to regular physical activity. Med Sci Sports Exerc. 2001;33(S6):S446-51.

36. Whelton SP, Chin A, Xin X, He J. Effect of aerobic exercise on blood pressure: a meta-analysis of randomized, controlled trials. Ann Intern Med. 2002;136(7):493-503.

37. Kodama S, Saito K, Tanaka S, et al. Cardiorespiratory fitness as a quantitative predictor of all-cause mortality and cardiovascular events in healthy men and women: A meta-analysis. JAMA. 2009; 301(19):2024-35.

38. Lee D-c, Sui X, Church TS, Lee I-M, Blair SN. Associations of cardiorespiratory fitness and obesity with risks of impaired fasting glucose and type 2 diabetes in men. Diabetes Care. 2009; 32(2):257-262. 